\title{
A force-free field with constant alpha in an oblate cylinder: A generalization of the Lundquist solution
}

\author{
M. Vandas ${ }^{1}$ and E. P. Romashets ${ }^{2}$ \\ 1 Astronomical Institute, Academy of Sciences, Boční II 1401, 14131 Praha 4, Czech Republic \\ e-mail: vandas@ig.cas.cz \\ 2 Institute of Terrestrial Magnetism, Ionosphere, and Radio Wave Propagation, Russian Academy of Sciences, Troitsk, \\ Moscow Region, 142190, Russia \\ e-mail: romash@izmiran.rssi.ru
}

Received 10 September 2002 / Accepted 30 October 2002

\begin{abstract}
A force-free magnetic field with constant alpha for a circular cylindrical flux rope (Lundquist solution) is widely used to describe the magnetic field configuration in interplanetary flux ropes. Observations as well as MHD simulations indicate that interplanetary flux ropes are not circular but have an oblate shape. Here we present an analytical solution for a force-free magnetic field with constant alpha in an elliptic flux rope which may be regarded as a direct generalization of the Lundquist solution. An alternative simpler solution for a force-free magnetic field with constant alpha in an oblate flux rope is discussed.
\end{abstract}

Key words. magnetic fields - Sun: coronal mass ejections (CMEs)

\section{Introduction}

A magnetic force-free field with constant alpha fulfills the equation

$\operatorname{rot} \boldsymbol{B}=\alpha \boldsymbol{B}$,

where $\alpha \neq 0$ is a constant. From a mathematical point of view, the force-free field is the simplest configuration after potential (current-free) solutions (potential solutions have $\alpha=0$ in (1)).

Lundquist (1950) has found a solution of (1) in the cylindrical geometry, which reads

$B_{r}=0$,

$B_{\varphi}=B_{0} J_{1}(\alpha r)$,

$B_{Z}=B_{0} J_{0}(\alpha r)$,

where $J_{n}$ are the Bessel functions. This solution is frequently used for a description of the magnetic field configuration in interplanetary flux ropes, including the most pronounced ones, viz. magnetic clouds (Burlaga 1988; Lepping et al. 1990). Due to its propagation in the diverging solar wind and due to its often higher velocity that the ambient solar wind, an interplanetary flux rope can hardly have a circular cross section, even if it had one initially in the solar corona (Klimchuk et al. 2000). Magnetohydrodynamic numerical simulations

Send offprint requests to: $\mathrm{M}$. Vandas,

e-mail: vandas@ig.cas.cz
(Vandas et al. 1995, 2002a; Cargill et al. 1996) show that propagating flux ropes will have oblate shapes. Mulligan \& Russell (2001) concluded from multispacecraft observations that magnetic clouds must be very oblate and they suggested a solution of a magnetic field in an oblate cylinder, which is not force-free (another solution, in an elliptical cylinder, which is also not force-free, but with a constant current, can be found in Pneuman 1983). We present here an analytical solution of a force-free field with constant alpha for an oblate flux rope, which may be regarded as a direct generalization of the Lundquist solution.

\section{Solution for an elliptic cylinder}

\subsection{Basic equation}

We solve Eq. (1) in the elliptic cylindrical coordinates $u, v$, and $Z$. These coordinates are defined by

$x=c \cosh u \cos v$,

$y=c \sinh u \sin v$,

$z=Z$,

where $c=\sqrt{a^{2}-b^{2}}, u \in\langle 0, \infty)$, and $v \in\langle 0,2 \pi)$. Contours with $u=$ const. are ellipses. The generating ellipse $\left(u=u_{0}\right)$ with semimajor axis $a$ and semiminor axis $b$ has

$\cosh u_{0}=a / c$ 
and its foci are at the points $( \pm c, 0)$ in the $x y$ plane. The Lamé coefficients are $h_{u}=h_{v}=c \sqrt{\cosh ^{2} u-\cos ^{2} v}$ and $h_{Z}=1$.

Equation (1) in elliptic cylindrical coordinates has the components

$$
\begin{aligned}
& \frac{1}{h_{u}} \frac{\partial B_{Z}}{\partial v}=\alpha B_{u}, \\
& -\frac{1}{h_{u}} \frac{\partial B_{Z}}{\partial u}=\alpha B_{v}, \\
& \frac{1}{h_{u}^{2}}\left[\frac{\partial}{\partial u}\left(h_{u} B_{v}\right)-\frac{\partial}{\partial v}\left(h_{u} B_{u}\right)\right]=\alpha B_{Z},
\end{aligned}
$$

if we assume that the searched magnetic field does not depend on $Z$.

A substitution for $B_{u}$ and $B_{v}$ from (9)-(10) into (11) yields the equation for $B_{Z}$

$$
\frac{\partial^{2} B_{Z}}{\partial u^{2}}+\frac{\partial^{2} B_{Z}}{\partial v^{2}}=-\alpha^{2} c^{2}\left(\cosh ^{2} u-\cos ^{2} v\right) B_{Z}
$$

From the expected behaviour of the magnetic field inside a flux rope, viz. that projections of magnetic field lines to the $x y$ plane will coincide or will be similar to ellipses and symmetric in quadrants of the $x y$ plane, these conditions follow

$$
\begin{aligned}
& B_{x}(x, 0, z)=0, \quad B_{y}(-x, 0, z)=-B_{y}(x, 0, z), \\
& B_{y}(0, y, z)=0, \quad B_{x}(0,-y, z)=-B_{x}(0, y, z) .
\end{aligned}
$$

\subsection{Solution}

We shall assume that the flux rope has the shape of the generating ellipse. We shall solve (12) by a method of separation of variables and denote

$\varepsilon=(\alpha c)^{2}$.

Let us assume that

$B_{Z}=B_{0} U_{\varepsilon}(u) V_{\varepsilon}(v)$,

where $U_{\varepsilon}(u)$ and $V_{\varepsilon}(v)$ are new functions (depending implicitly also on $\varepsilon$, which is stressed by the subscript), $V_{\varepsilon}(v)$ is periodic with the period $\pi$. We can put without loss of generality

$U_{\varepsilon}(0)=1, \quad V_{\varepsilon}(0)=1$.

The other components (9)-(10) are expressed

$B_{u}=\frac{1}{\alpha h_{u}} \frac{\partial B_{Z}}{\partial v}=\frac{B_{0}}{\alpha h_{u}} U_{\varepsilon}(u) V_{\varepsilon}^{\prime}(v)$,

$B_{v}=-\frac{1}{\alpha h_{u}} \frac{\partial B_{Z}}{\partial u}=-\frac{B_{0}}{\alpha h_{u}} U_{\varepsilon}^{\prime}(u) V_{\varepsilon}(v)$.

At the boundary, $u=u_{0}$, it must hold for all $v$

$B_{u}\left(u_{0}, v\right)=\frac{B_{0}}{\alpha h_{u}} U_{\varepsilon}\left(u_{0}\right) V_{\varepsilon}^{\prime}(v)=0$,

therefore

$U_{\varepsilon}\left(u_{0}\right)=0$.

This condition yields $B_{Z}=0$ (cf. (16)) at the boundary.
Let us examine the conditions (13)-(14). $B_{y}$ in the $x=0$ plane must fulfill

$B_{y}(0, y, z)=0=\left.B_{u}\right|_{v=\pi / 2,3 \pi / 2}=\frac{B_{0}}{\sqrt{\varepsilon} \cosh u} U_{\varepsilon}(u) V_{\varepsilon}^{\prime}\left(\frac{\pi}{2}\right)$

therefore

$V_{\varepsilon}^{\prime}\left(\frac{\pi}{2}\right)=0$,

taking into account the periodicity of $V_{\varepsilon}$. The situation in the $y=0$ plane is more complicated. For $x>c$ we have

$B_{x}(x, 0, z)=0=\left.B_{u}\right|_{v=0}=\frac{B_{0}}{\sqrt{\varepsilon} \sinh u} U_{\varepsilon}(u) V_{\varepsilon}^{\prime}(0)$,

therefore

$V_{\varepsilon}^{\prime}(0)=0$

For $0<x<c$ we have

$B_{x}(x, 0, z)=0=-\left.B_{v}\right|_{u=0}=\frac{B_{0}}{\sqrt{\varepsilon} \sin v} U_{\varepsilon}^{\prime}(0) V_{\varepsilon}(v)$,

therefore

$U_{\varepsilon}^{\prime}(0)=0$.

The other conditions (13)-(14) are fulfilled because of the periodicity of $V_{\varepsilon}$. It is $B_{x}=0$ at the foci and $B_{y}$ must be continuous there. This will yield an additional condition for the functions $U_{\varepsilon}(u)$ and $V_{\varepsilon}(v)$. Let us calculate the limits when approaching the right focus:

$\lim _{x \rightarrow c+} B_{y}(x, 0, z)=\left.\lim _{u \rightarrow 0} B_{v}\right|_{v=0}=-\lim _{u \rightarrow 0} \frac{B_{0}}{\sqrt{\varepsilon} \sinh u} U_{\varepsilon}^{\prime}(u)$,

$\lim _{x \rightarrow c-} B_{y}(x, 0, z)=\left.\lim _{v \rightarrow 0} B_{u}\right|_{u=0}=\lim _{v \rightarrow 0} \frac{B_{0}}{\sqrt{\varepsilon} \sin v} V_{\varepsilon}^{\prime}(v)$

(the relationships (17) were used). These limits must be equal, so

$\lim _{u \rightarrow 0} \frac{U_{\varepsilon}^{\prime}(u)}{\sinh u}+\lim _{v \rightarrow 0} \frac{V_{\varepsilon}^{\prime}(v)}{\sin v}=0$.

Equation (12) splits into two equations when introducing (16)

$U_{\varepsilon}^{\prime \prime}-\left(K_{\varepsilon}-\varepsilon \cosh ^{2} u\right) U_{\varepsilon}=0$,

$V_{\varepsilon}^{\prime \prime}+\left(K_{\varepsilon}-\varepsilon \cos ^{2} v\right) V_{\varepsilon}=0$

where $K_{\varepsilon}$ is a constant used in the separation of variables method. Equation (27) is a Mathieu equation (Whittaker \& Watson 1997; Kuznetsov 1965). The solution $V_{\varepsilon}$ is periodic only for a specific set of separation constants $K_{\varepsilon}$ which depend on $\varepsilon$ (that is why there is a subscript $\varepsilon$ in $K_{\varepsilon}$ ). The solution, which fulfils conditions (17) and (22)-(23), is

$V_{\varepsilon}(v)=\frac{\mathrm{ce}_{0}(v,-\varepsilon / 32)}{\mathrm{ce}_{0}(0,-\varepsilon / 32)}$,

where $\operatorname{ce}_{0}(v, q)$ is the even Mathieu function of zero order. It tends to 1 when $q \rightarrow 0$, therefore $V_{\varepsilon} \rightarrow 1$ when oblateness disappears and we have no angular dependence of the solution for a cylindrical case, as expected. 
The substitution $\tilde{u}=i u$ ( $i$ is an imaginary unit) converts (26) exactly into the form of (27), therefore the solution is

$U_{\varepsilon}(u)=\frac{\operatorname{ce}_{0}(i u,-\varepsilon / 32)}{\operatorname{ce}_{0}(0,-\varepsilon / 32)}=\frac{\operatorname{ceh}_{0}(u,-\varepsilon / 32)}{\operatorname{ce}_{0}(0,-\varepsilon / 32)}$,

where $\operatorname{ceh}_{0}(u, q)$ is the modified even Mathieu function of zero order. All conditions (17) and (22)-(25) are fulfilled.

$K_{\varepsilon}$ for a given $\varepsilon$ is expressed by an infinite fraction (Kuznetsov 1965)

$K_{\varepsilon}=\frac{\varepsilon}{2}+\frac{\varepsilon^{2}}{8} \frac{\Delta_{2}}{\Delta_{1}}$

$\frac{\Delta_{n}}{\Delta_{n+1}}=K_{\varepsilon}-\frac{\varepsilon}{2}-4 n^{2}-\frac{\varepsilon^{2}}{16} \frac{\Delta_{n+2}}{\Delta_{n+1}}$.

For the calculation of $K_{\varepsilon}$ the fraction is cut at some level and (30)-(31) then represent an implicit equation for $K_{\varepsilon}$ (the first root), which can be solved, e.g., by a method of bisections (for smaller $\varepsilon$ an iteration method also works).

For calculation purposes we derive expressions for the functions $U_{\varepsilon}(u)$ and $V_{\varepsilon}(v)$ in the form of a series. The substitution $w=\sin ^{2} v$ changes (27) (the prime is now a derivative by $w$ ) into

$4 w(1-w) V^{\prime \prime}+2(1-2 w) V^{\prime}+\left(K_{\varepsilon}-\varepsilon+\varepsilon w\right) V=0$.

A solution of (32) is

$V_{\varepsilon}(w)=\sum_{n=0}^{\infty} a_{n} w^{n}$.

Substituting (33) into (32) and equating the coefficients at $w^{n}$, we get

$a_{1}=-\frac{\left(K_{\varepsilon}-\varepsilon\right) a_{0}}{2}$

$a_{n}=\frac{\left[4(n-1)^{2}-K_{\varepsilon}+\varepsilon\right] a_{n-1}-\varepsilon a_{n-2}}{2 n(2 n-1)}$.

The condition (17) yields

$a_{0}=1$

and the conditions (22)-(23) are automatically fulfilled due to the selected substitution. A profile of the function $V_{\varepsilon}$ is shown in Fig. 1 for $\varepsilon=25 ; K_{\varepsilon} \approx 4.732952$ in this case.

Equation (26) will be solved in a similar way. The substitution $s=\tanh ^{2} u$ converts it (the prime is now a derivative by $s$ ) into

$4 s(1-s)^{3} U^{\prime \prime}+2(1-s)^{2}(1-3 s) U^{\prime}-\left(K_{\varepsilon}-\varepsilon-K_{\varepsilon} s\right) U=0$.

A solution of (34) is

$U_{\varepsilon}(s)=\sum_{n=0}^{\infty} b_{n} s^{n}$.

Substituting (35) into (34) we get

$b_{1}=\frac{\left(K_{\varepsilon}-\varepsilon\right) b_{0}}{2}, \quad b_{2}=\frac{\left(10+K_{\varepsilon}-\varepsilon\right) b_{1}-K_{\varepsilon} b_{0}}{12}$,

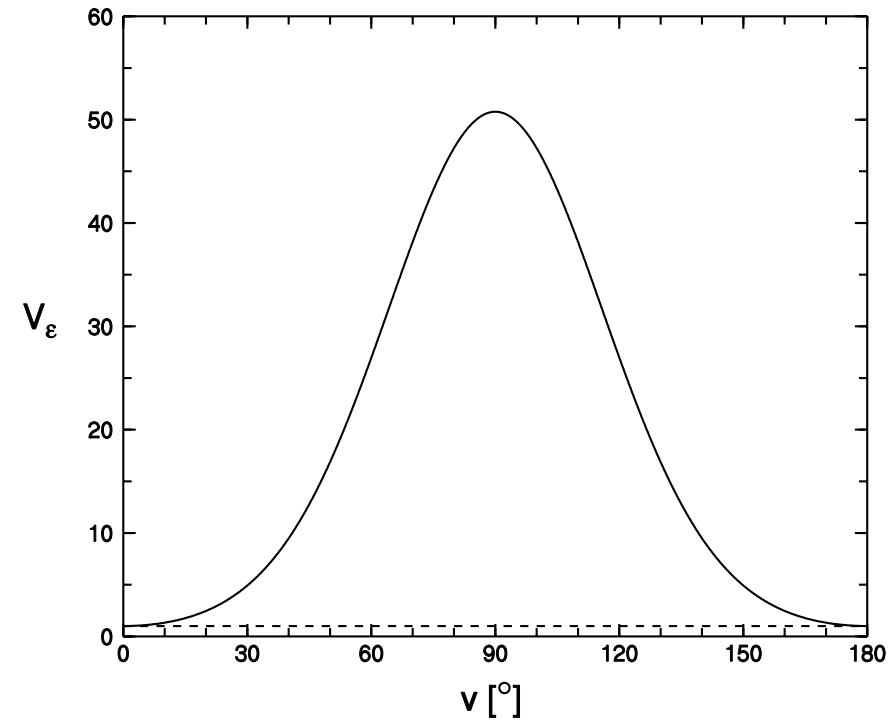

Fig. 1. The function $V_{\varepsilon}$ for $\varepsilon=25$ (this corresponds approximately to $a / b \approx 3$ ). It deviates strongly from $V_{\varepsilon}=1$ for zero oblateness, drawn by the dashed line.

$$
\begin{aligned}
b_{n}= & \left\{\left[2(n-1)(6 n-7)+K_{\varepsilon}-\varepsilon\right] b_{n-1}\right. \\
& -\left[2(n-2)(6 n-11)+K_{\varepsilon}\right] b_{n-2} \\
& \left.+2(n-3)(2 n-5) b_{n-3}\right\} /[2 n(2 n-1)] .
\end{aligned}
$$

The condition (17) yields

$b_{0}=1$

and the condition (24) is automatically fulfilled due to the selected substitution. The derived series also meet the condition (25).

A profile of the function $U_{\varepsilon}$ is shown in Fig. 2 for $\varepsilon=25$; the same as used in Fig. 1, so the functions $U_{\varepsilon}$ and $V_{\varepsilon}$ from Figs. 1, 2 determine a particular solution of the magnetic field. The function $U_{\varepsilon}$ has roots and its first root (labelled by $u_{0}$ in Fig. 2) determines an elliptic boundary of the flux rope (21), and hence its oblateness by (8).

\subsection{Solution for a given oblateness}

Let us have a given ellipse with $a$ and $b$; we shall construct a solution for a flux rope having the shape of this ellipse. More specifically, the boundary of the flux rope will be defined by $B_{Z}=0$ and will coincide with this ellipse. The semiaxes $a$ and $b$ determine $u_{0}$ by (8) and we must find an appropriate function $U_{\varepsilon}$ for which the first root is $u_{0}$. Equation (21) is an implicit equation for $\varepsilon$ and can be solved by the method of bisections. A given $\varepsilon$ determines $K_{\varepsilon}$ by (30)-(31) and then $U_{\varepsilon}$ by (35). The condition (21) is checked etc. The final $\varepsilon$ defines $\alpha$ by (15). For a numerical 


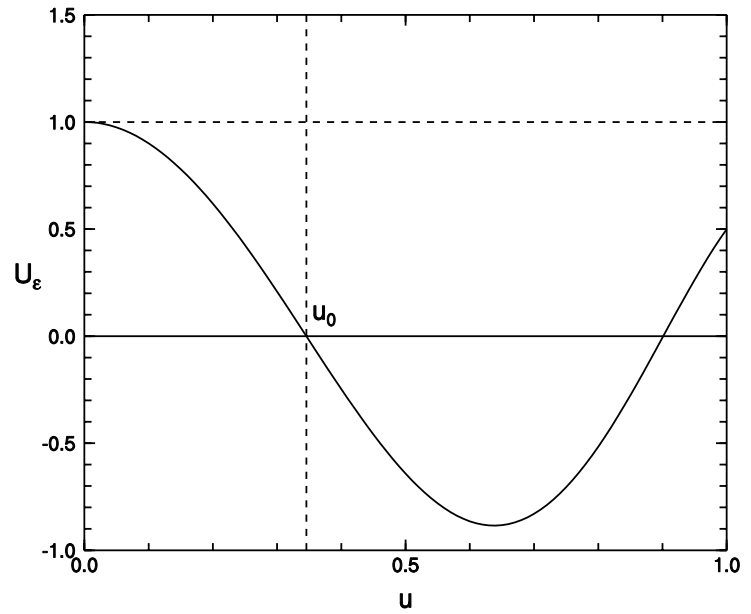

Fig. 2. The function $U_{\varepsilon}$ for $\varepsilon=25$. Its first root is denoted by $u_{0}$ and stressed by the dashed vertical line. When oblateness goes to zero, $u_{0}$ shifts to larger values and the profile of the function $U_{\varepsilon}$ for $u \ll u_{0}$ becomes closer and closer to $U_{\varepsilon}=1$, drawn by the dashed horizontal line.

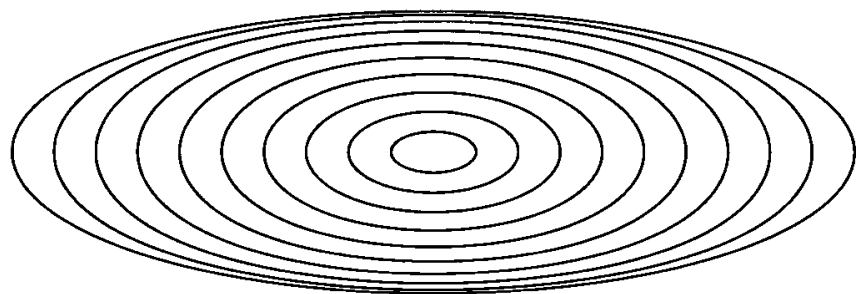

Fig. 3. Magnetic field lines for case $a / b=3$. The flux rope boundary is the generating ellipse.

solution it is useful to use a table of pre-calculated values:

$\begin{array}{lcrc}a / b & u_{0} & \varepsilon & K_{\varepsilon} \\ 1.25 & 1.098612 & 2.663889 & 1.119968 \\ 1.5 & 0.804719 & 5.197484 & 1.869170 \\ 2 & 0.549306 & 10.700177 & 2.962558 \\ 3 & 0.346574 & 24.864983 & 4.719335 \\ 4 & 0.255413 & 43.797785 & 6.357117 \\ 5 & 0.202733 & 67.633761 & 7.965562 \\ 6 & 0.168236 & 96.386108 & 9.560726 .\end{array}$

Figure 3 shows magnetic field lines for our solution when $a / b=3$. The largest oval is the boundary of the flux rope and it is an ellipse, which coincides with the generating ellipse. Because the magnetic field components do not depend on $Z$ (i.e., $z$ ), magnetic field lines are defined by conditions $B_{Z}=$ const., as can be demonstrated by the following calculation

$$
\begin{aligned}
\boldsymbol{B} \cdot \operatorname{grad} B_{Z} & =\frac{B_{u}}{h_{u}} \frac{\partial B_{Z}}{\partial u}+\frac{B_{v}}{h_{v}} \frac{\partial B_{Z}}{\partial v} \\
& =\frac{1}{h_{u}^{2}} \frac{\partial B_{Z}}{\partial v} \frac{\partial B_{Z}}{\partial u}-\frac{1}{h_{u}^{2}} \frac{\partial B_{Z}}{\partial u} \frac{\partial B_{Z}}{\partial v}=0,
\end{aligned}
$$

where relationships (9)-(10) were used. Magnetic field lines in Fig. 3 are also contour lines of $B_{Z}$ and the largest contour has $B_{Z}=0$. So also another surface $B_{Z}=$ const. may be taken as a boundary of a flux rope, but its cross section will not be an exact ellipse. Figure 4 shows deviations of boundaries of flux ropes smaller than the generating ellipse from elliptic shapes.

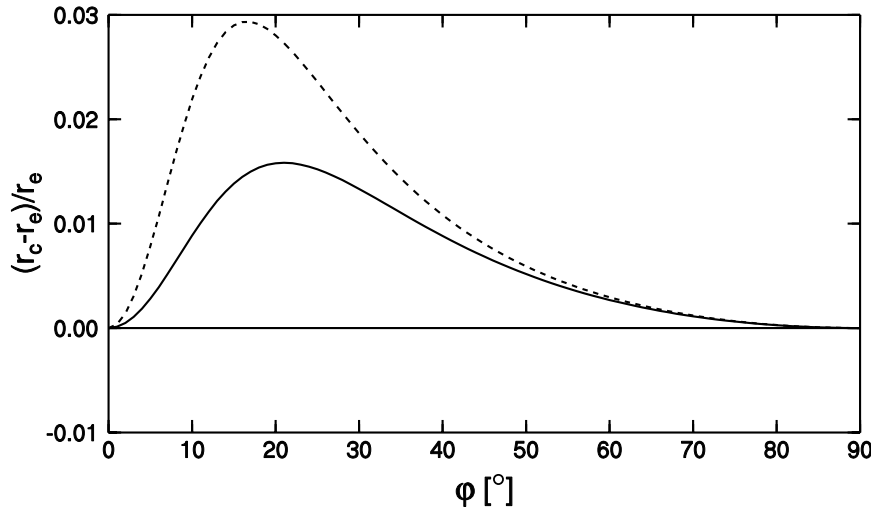

Fig. 4. Deviation of inner magnetic field line projections (on the $z=0$ plane) from ellipses. Let the projection of a magnetic field line crosses the $x$ axis at $a^{\prime}$ and the $y$ axis at $b^{\prime}$. This determines the ellipse with semiaxes $a^{\prime}$ and $b^{\prime}$ and its deviation from the projection of the magnetic field line is shown; $r_{\mathrm{c}}$ is the distance of the projection of the magnetic field line from the centre for a given polar angle $\varphi$, and $r_{\mathrm{e}}$ is a similar distance for the ellipse. The solid line is for case $a / b=3$, the dashed line for case $a / b=4 ; a^{\prime}=3 / 4 a$.

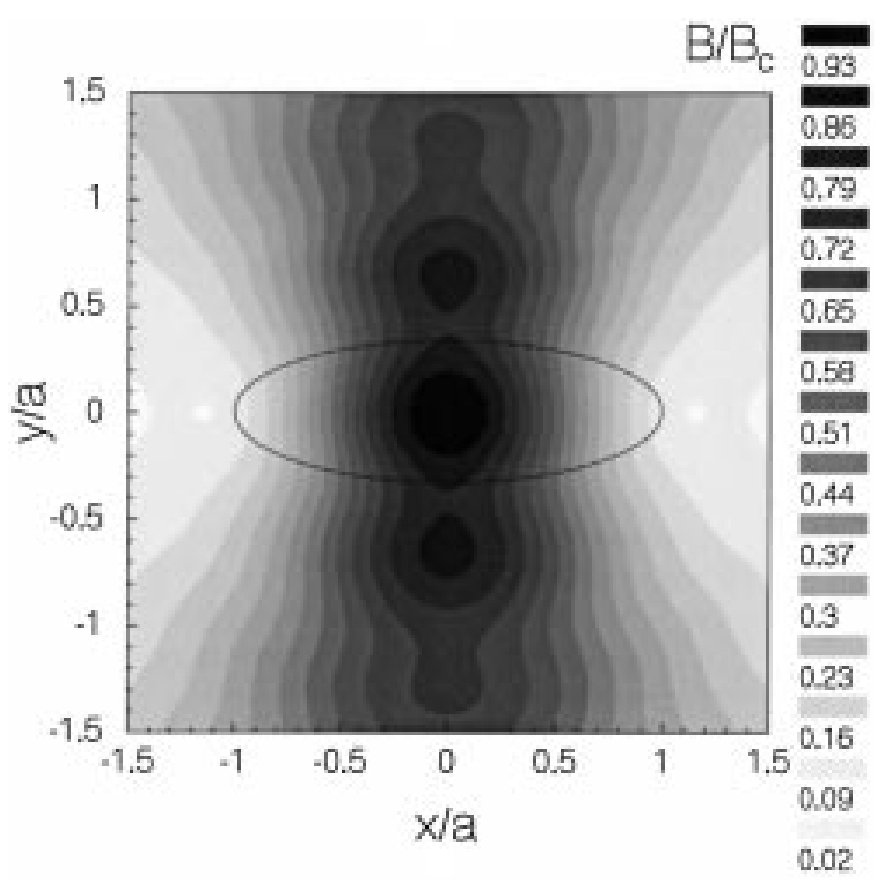

Fig. 5. Magnetic field magnitude distribution inside and around an elliptic flux rope $(a / b=3)$. The black oval shows the flux rope boundary (generating ellipse). The magnetic field magnitude $B$ is scaled by the central (maximum) magnitude value $B_{\mathrm{c}}$.

So projections of magnetic field lines are not strict ellipses; their deviations from elliptic shapes are more pronounced for larger oblateness and for "middle" regions inside the generating ellipse, but they are quite small.

The magnetic field magnitude distribution is shown in Fig. 5. While projections of magnetic field lines are oblate ovals, the contours of the field magnitude are prolate. A similar distribution can be found in flux ropes from MHD simulations (e.g., Vandas et al. 2002b). 


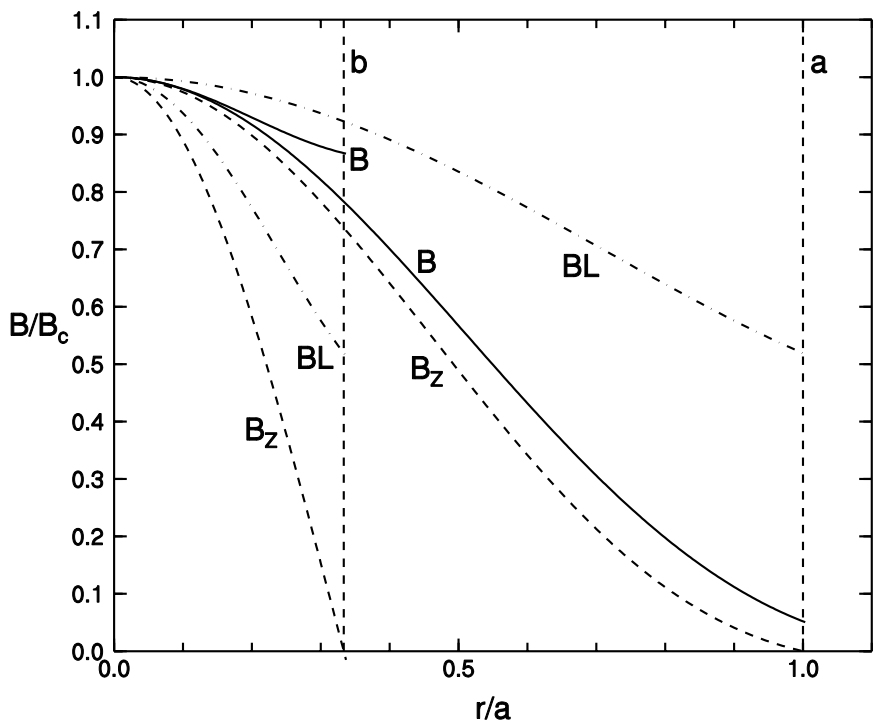

Fig. 6. Profiles of the magnetic field magnitude (labeled B, solid lines) along the semiaxes $a$ and $b$ in an elliptic flux rope when $a / b=3$ (case shown in Figs. 3 and 5). For comparison profiles of the magnetic field magnitude for the corresponding Lundquist solution (labeled BL, dash-dotted lines) were added. The dashed lines give profiles of the $B_{Z}$ component in the elliptic flux rope. The magnetic field profiles are scaled by the central (maximum) magnitude value $B_{\mathrm{c}}$.

The magnetic field strength falls off more slowly along the shorter semiaxis $b$ than along the longer one $a$. A similar statement is true when comparing with the Lundquist solution for a circular cylinder (Fig. 6). The magnetic field strength falls off more slowly along the shorter semiaxis $b$ than in a circular flux rope with radius $b$ and the opposite is true for the longer semiaxis $a$ in comparison with a circular flux rope with radius $a$ (the boundary of a circular flux rope is also defined by $B_{Z}=0$ ). The differences are quite remarkable.

\subsection{Correspondence to the Lundquist solution}

A correspondence to the Lundquist solution can be demonstrated in the following way. For a nearly circular cylinder $c \approx 0$ holds $(\varepsilon \approx 0)$. For $x$ and $y$ to have $O(1)$ values, $u$ must be large, i.e., $u \gg 1, \cosh u \gg 1, \sinh u \approx \cosh u$. The cylindrical coordinates are approximately $r \approx c \cosh u \approx c \sinh u, \varphi \approx v$. The quantities $K_{\varepsilon}$ (Whittaker \& Watson 1997), $V_{\varepsilon}(33)$, and $U_{\varepsilon}$ (a series in the Bessel functions $J_{n}$ for the function ceh $_{0}$ was used, Kuznetsov 1965) for small $\varepsilon$ are

$$
\begin{aligned}
K_{\varepsilon}= & \frac{\varepsilon}{2}-\frac{\varepsilon^{2}}{32}+\ldots, \\
V_{\varepsilon}= & 1+\left(\frac{\varepsilon}{4}+\frac{\varepsilon^{2}}{64}\right) \sin ^{2} v+\frac{\varepsilon^{2}}{64} \sin ^{4} v+\ldots, \\
U_{\varepsilon}= & J_{0}(\sqrt{\varepsilon} \sinh u)-\frac{\varepsilon}{8} J_{2}(\sqrt{\varepsilon} \sinh u) \\
& +\frac{\varepsilon^{2}}{512} J_{4}(\sqrt{\varepsilon} \sinh u)+\ldots
\end{aligned}
$$

The magnetic field components in the cylindrical system (cf. (2)-(4)) are

$$
\begin{aligned}
& B_{Z}=\lim _{c \rightarrow 0} B_{0} U_{\varepsilon} V_{\varepsilon}=\lim _{c \rightarrow 0} B_{0} J_{0}(\alpha c \sinh u)=B_{0} J_{0}(\alpha r), \\
& B_{r}=\lim _{c \rightarrow 0} B_{u}=\lim _{c \rightarrow 0} \frac{\alpha c B_{0}}{2 \cosh u} J_{0}(\alpha c \sinh u) \sin v \cos v=0, \\
& B_{\varphi}=\lim _{c \rightarrow 0} B_{v}=\lim _{c \rightarrow 0} B_{0} J_{1}(\alpha c \sinh u)=B_{0} \mathrm{~J}_{1}(\alpha r) .
\end{aligned}
$$

\subsection{An alternative solution for smaller oblatenesses}

We also examined another solution which yields an oblate flux rope, namely a sum of two Lundquist solutions with different centres. If we have two force-free solutions with the same $\alpha$, then their sum is a force-free solution with $\alpha$, too, due to the linearity of Eq. (1). Formally this solution can be derived from (12). Let us make a substitution of variables, $s=\alpha c(\cosh u-\cos v)$, and assume that $B_{Z}$ depends on $s$ only, $B_{Z}=B_{0} q(s)$, where $q(s)$ is an unknown function. Equation (12) is very much simplified with such a $B_{Z}$ and one gets a Bessel equation

$q^{\prime \prime}+\frac{q^{\prime}}{s}+q=0$

with the solution $q(s)=J_{0}(s)$. The same procedure can be repeated for $\tilde{s}=\alpha c(\cosh u+\cos v), B_{Z}=B_{0} \tilde{q}(\tilde{s})$, with the solution $\tilde{q}(\tilde{s})=J_{0}(\tilde{s})$. So the general solution of (12) is a linear combination of the two above given solutions. But the condition (14) fulfills only the combination

$$
\begin{aligned}
B_{Z}=\frac{B_{0}}{2} & \left\{J_{0}[\alpha c(\cosh u+\cos v)]\right. \\
& \left.+J_{0}[\alpha c(\cosh u-\cos v)]\right\} .
\end{aligned}
$$

In fact, the two separate solutions represent the exact Lundquist solutions (2)-(4) in the elliptic cylindrical coordinates for cylindrical flux ropes centred in one and the other focus of the generating ellipse, respectively.

The other magnetic field components follow from (9) and (10):

$$
\begin{aligned}
B_{u}= & \frac{B_{0} \sin v}{2 \sqrt{\cosh ^{2} u-\cos ^{2} v}}\left\{J_{1}[\alpha c(\cosh u+\cos v)]\right. \\
& \left.-J_{1}[\alpha c(\cosh u-\cos v)]\right\} \\
B_{v}= & \frac{B_{0} \sinh u}{2 \sqrt{\cosh ^{2} u-\cos ^{2} v}}\left\{J_{1}[\alpha c(\cosh u+\cos v)]\right. \\
& \left.+J_{1}[\alpha c(\cosh u-\cos v)]\right\}
\end{aligned}
$$

When the oblateness disappears, the foci move to the centre and the solution becomes the Lundquist solution. Formally

$$
\begin{aligned}
& B_{r} \approx B_{u} \approx \frac{B_{0} \sin v}{2 \cosh u}\left[J_{1}(\alpha c \cosh u)-J_{1}(\alpha c \cosh u)\right]=0, \\
& B_{\varphi} \approx B_{v} \approx \frac{B_{0} \sinh u}{2 \cosh u}\left[J_{1}(\alpha c \cosh u)+J_{1}(\alpha c \cosh u)\right] \\
& \approx B_{0} J_{1}(\alpha r) \\
& B_{Z} \approx \frac{B_{0}}{2}\left[J_{0}(\alpha c \cosh u)+J_{0}(\alpha c \cosh u)\right] \approx B_{0} J_{0}(\alpha r) \\
& \text { (cf. (2)-(4)). }
\end{aligned}
$$




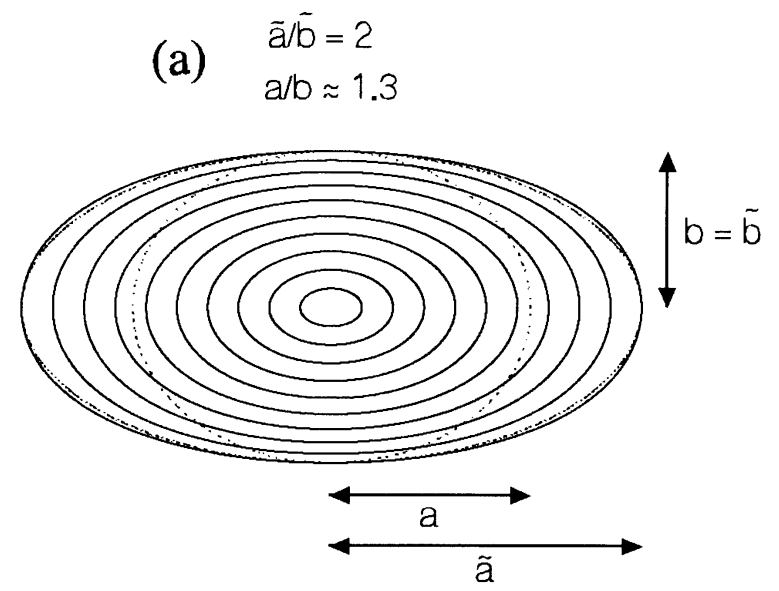

(b) $\tilde{a} / \tilde{b}=3.1938$

$\mathrm{a} / \mathrm{b} \approx 1.57$

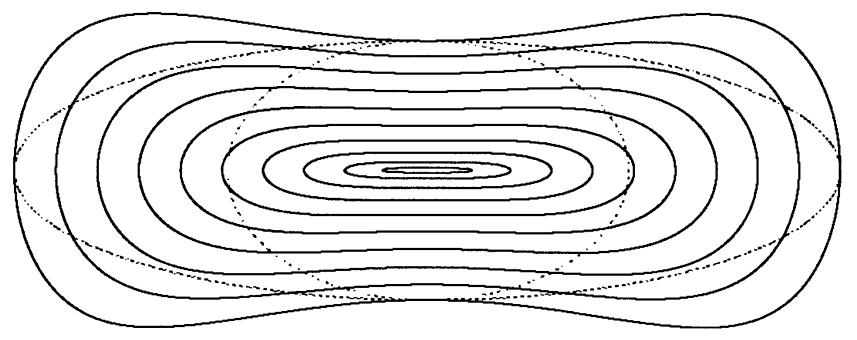

(c) $\tilde{a} / \tilde{b}=3.5$

$a / b \approx 1.69$

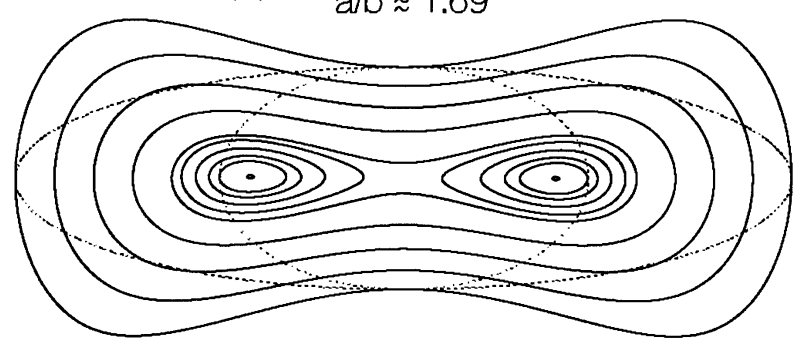

Fig. 7. Magnetic field lines (thick lines) for three different values of the oblateness $a / b$ of flux ropes. The longest magnetic field lines are flux rope boundaries where $B_{Z}=0$. The thin dashed lines are generating ellipses given by semiaxes $a$ and $b$, the thin solid lines are ellipses given by semiaxes $\tilde{a}$ and $\tilde{b}$.

The magnetic field lines of this solution are not strict ellipses, as can be seen from Fig. 7. Their deviation from an elliptical shape is more pronounced for larger oblateness (compare Figs. 7a and 7b). As has been stated, magnetic field lines were defined by conditions $B_{Z}=$ const., therefore magnetic field lines in Fig. 7 are also contour lines of $B_{Z}$ and the largest contours have $B_{Z}=0$.

As is usual for a cylindrical flux rope, we shall assume $B_{Z}=0$ on our flux rope boundary. The boundary does not coincide with a generating ellipse (nor another ellipse) and we shall determine $c$ for a given "elliptical" flux rope with semiaxes $\tilde{a}$ and $\tilde{b}$ in a sense that the rope boundary crosses the $x$ axis at the distance $\tilde{a}$ and the $y$ axis at the distance $\tilde{b}$. We may set $\tilde{b}=b$. The denomination is illustrated in Fig. 7a.

From $B_{Z}=0$ at the point $(0, \tilde{b})=(0, b)$ in the $x y$ plane, i.e., $v=\pi / 2$, we have a condition $J_{0}(\alpha a)=0$, i.e., $\alpha=r_{1} / a$, where $r_{1}$ is the first root of $J_{0}(r)\left(r_{1} \approx 2.4048\right)$. From $B_{Z}=0$ at the point $(\tilde{a}, 0)$ in the $x y$ plane, i.e., $v=0$, we have a condition

$$
\begin{aligned}
& \mathrm{J}_{0}\left[r_{1} \sqrt{1-\lambda^{\prime 2}}\left(\frac{1}{\lambda \sqrt{\lambda^{\prime 2}-1}}-1\right)\right] \\
& +\mathrm{J}_{0}\left[r_{1} \sqrt{1-\lambda^{\prime 2}}\left(\frac{1}{\lambda \sqrt{\lambda^{\prime 2}-1}}+1\right)\right]=0,
\end{aligned}
$$

where $\lambda=\tilde{b} / \tilde{a}$ and $\lambda^{\prime}=b / a$. This is an equation for $\lambda^{\prime}$ and $c=$ $\tilde{b} \sqrt{\lambda^{\prime-2}-1}$. The $B_{Z}=0$ boundary is very close to an ellipse for smaller oblateness $\tilde{a} / \tilde{b}<2$.

We cannot get an arbitrary large oblateness for a single flux rope; there is a limit above which magnetic field lines split into two flux ropes embedded into a larger closed common structure ("double flux rope" - see Fig. 7c). Such a behaviour is obvious, because the solution is the sum of two Lundquist solutions; for larger oblatenesses the foci are sufficiently distant for each Lundquist solution "to manifest its identity". The limiting oblateness can be found by examining the $B_{Z}$ profile along the $\tilde{a}$ semiaxis. For smaller oblateness this profile has a maximum at the centre, but for larger ones the maximum is shifted and there is a local minimum (depression) at the centre. We have $u=0$ and $v \approx \pi / 2$ near the centre along the $a$ semiaxis:

$B_{Z}(v)=\frac{B_{0}}{2}\left\{J_{0}[\alpha c(1+\cos v)]+J_{0}[\alpha c(1-\cos v)]\right\}$.

The first derivative of $B_{Z}(v)$,

$$
\begin{aligned}
B_{Z}^{\prime}(v)= & \frac{B_{0}}{2} \alpha c \sin v\left\{J_{1}[\alpha c(1+\cos v)]\right. \\
& \left.-J_{1}[\alpha c(1-\cos v)]\right\},
\end{aligned}
$$

is zero at the centre $(v=\pi / 2)$, indicating a presence of an extremum. The type of the extremum is determined by a sign of the second derivative, which at the centre reads $B_{Z}^{\prime \prime}\left(\frac{\pi}{2}\right)=$ $-B_{0} \alpha^{2} c^{2} J_{1}^{\prime}(\alpha c)$. At the limiting oblateness $B_{Z}^{\prime \prime}$ changes its sign from minus ( $B_{Z}$ has a local maximum) to plus ( $B_{Z}$ has a local minimum), i.e., $B_{Z}^{\prime \prime}(\pi / 2)=0$ must hold, which reduces to a condition $J_{1}^{\prime}(\alpha c)=0$. The relationship $\alpha c=r_{1} \sqrt{1-\lambda^{\prime 2}}$ holds. Denoting $r_{2}$ the first root of $J_{1}^{\prime}(r), r_{2} \approx 1.8412$, we get a limiting oblateness of the generating ellipse, $\lambda_{m}^{\prime}=\sqrt{1-\left(r_{2} / r_{1}\right)^{2}} \approx$ 0.6433 . Substituting $\lambda_{m}^{\prime}$ into (43) yields the condition for the limiting oblateness, $\lambda_{m}$,

$J_{0}\left(\frac{\sqrt{r_{1}^{2}-r_{2}^{2}}}{\lambda_{m}}-r_{2}\right)+J_{0}\left(\frac{\sqrt{r_{1}^{2}-r_{2}^{2}}}{\lambda_{m}}+r_{2}\right)=0$.

Due to the oscillating nature of $J_{0}$ there are more solutions of (44). The $\lambda_{m}$ closest to 1 (the smallest oblateness) is $\lambda_{m} \approx$ 0.3131 , so for the limiting case $\tilde{a} / \tilde{b} \approx 3.1938$ holds. However, the shape of the flux rope is far from the ellipse given by $\tilde{a}$ and $\tilde{b}$ in this case and resembles a dumbbell (Fig. 7b). The limiting case splits the solution representing a single flux rope (Fig. 7a) and a double flux rope (Fig. 7c).

Figure 8 shows the magnetic field magnitude distribution inside and outside of a flux rope for the case displayed in Fig. 7a. The qualitative behaviour of this distribution is quite 


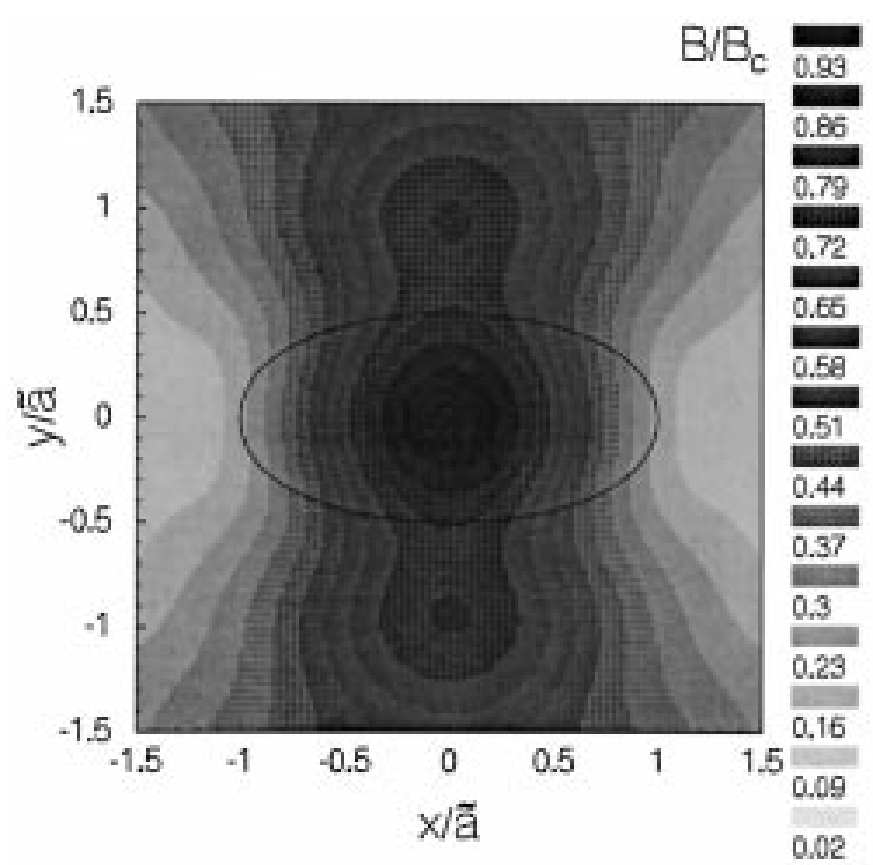

Fig. 8. Magnetic field magnitude distribution inside and around an oblate flux rope. The black oval shows the flux rope boundary where $B_{Z}=0(\tilde{a} / \tilde{b}=2)$. The magnetic field magnitude $B$ is scaled by the central (maximum) magnitude value $B_{\mathrm{c}}$.

similar to that of the solution for an elliptic flux rope given above. This similarity is also demonstrated in Fig. 9, where the magnetic field profiles are given for the two presented solutions. Therefore the second solution can be used for moderate oblatenesses. Its advantage is its simplicity.

\section{Conclusions}

We have found an analytical solution of a force-free magnetic field with constant alpha in an elliptic cylindrical flux rope. Magnetic field lines inside the flux rope are not strict elliptic helices. They are ellipses only on the boundary. The obtained solution represents a generalization of the Lundquist solution for a circular cylinder. We have also presented another solution for an oblate cylindrical flux rope, which can be used for smaller oblatenesses and is simpler.

Acknowledgements. This work was supported by grant A3003003 and project S1003006 from the Academy of Sciences of the Czech Republic, and by EU/INTAS/ESA grant 99-00727 and CDRF grant TGP-848.

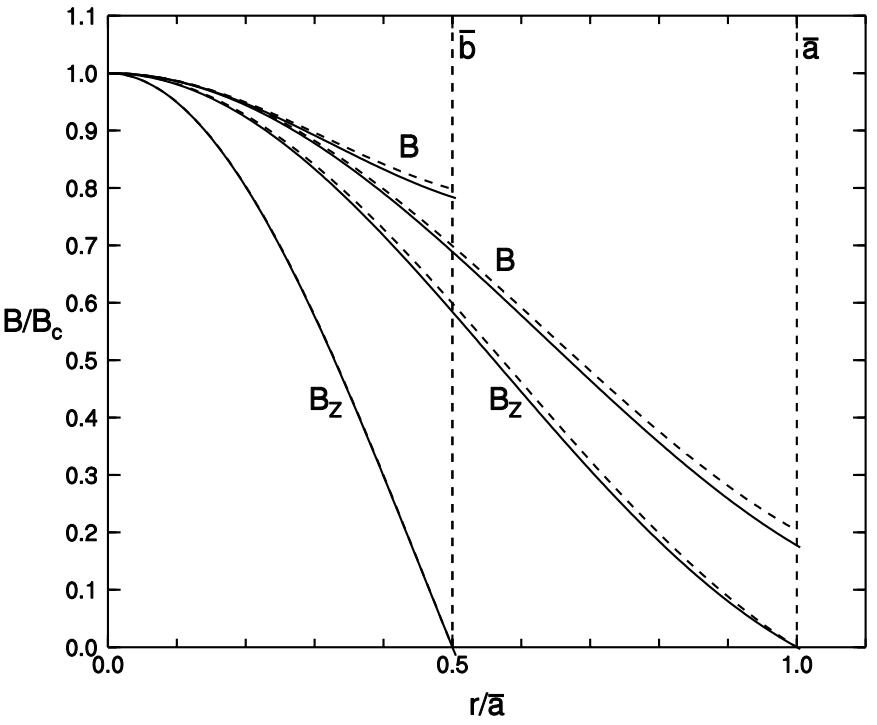

Fig. 9. Profiles of the magnetic field magnitude (labeled $B$ ) and the $B_{Z}$ component along the semiaxes $\bar{a}$ and $\bar{b}$ in an oblate flux rope when $\bar{a} / \bar{b}=2$. The solid lines are for the first solution (an exact elliptic cylinder, $\bar{a}=a, \bar{b}=b$ ), the dashed lines for the second solution $(\bar{a}=\tilde{a}, \bar{b}=\tilde{b})$. The magnetic field profiles are scaled by the central (maximum) magnitude value $B_{\mathrm{c}}$.

\section{References}

Burlaga, L. F. 1988, J. Geophys. Res., 93, 7217

Cargill, P. J., Chen, J., Spicer, D., \& Zalesak, S. T. 1996, J. Geophys. Res., 101, 4855

Klimchuk, J. A., Antiochos, S. K., \& Norton, D. 2000, ApJ, 542, 504

Kuznetsov, D. S. 1965, Special Functions (Vysshaya shkola, Moscow, in Russian)

Lepping, R. P., Jones, J. A., \& Burlaga, L. F. 1990, J. Geophys. Res., 95, 11957

Lundquist, S. 1950, Ark. Fys., 2, 361

Mulligan, T., \& Russell, C. T. 2001, J. Geophys. Res., 106, 10581

Pneuman, G. W. 1983, ApJ, 265, 468

Vandas, M., Fischer, S., Dryer, M., Smith, Z., \& Detman, T. 1995, J. Geophys. Res., 100, 12285

Vandas, M., Odstrčil, D., \& Watari, S. 2002a, J. Geophys. Res., 107, 10.1029/2001JA005068

Vandas, M., Watari, S., \& Geranios, A. 2002b, in AIP Conf. Proc., Solar Wind Ten (AIP, Woodbury, New York), to appear

Whittaker, E. T., \& Watson, G. N. 1997, A Course of Modern Analysis (Cambridge University Press, Cambridge) 